\title{
Responses of quark condensates to the chemical potential
}

\author{
O. Miyamura* ${ }^{1}$ S. Choe,${ }^{2}$ Y. Liu, ${ }^{1}$ T. Takaishi, ${ }^{3}$ and A. Nakamura ${ }^{4}$ \\ ${ }^{1}$ Dept. of Physics, Hiroshima University, Higashi-Hiroshima 739-8526, Japan \\ ${ }^{2}$ Dept. of Chemistry, Korea Advanced Institute of Science and Technology, Daejon 305-701, Korea \\ ${ }^{3}$ Hiroshima University of Economics, Hiroshima 731-01, Japan \\ ${ }^{4}$ IMC, Hiroshima University, Higashi-Hiroshima 739-8521, Japan
}

\begin{abstract}
The responses of quark condensates to the chemical potential, as a function of temperature $T$ and chemical potential $\mu$, are calculated within the Nambu-Jona-Lasinio (NJL) model. We compare our results with those from the recent lattice QCD simulations [QCD-TARO Collaboration, Nucl. Phys. B (Proc. Suppl.) 106, 462 (2002)]. The NJL model and lattice calculations show qualitatively similar behavior, and they will be complimentary ways to study hadrons at finite density. The behavior above $T_{c}$ requires more elaborated analyses.
\end{abstract}

PACS numbers: 12.39.-x

The variation of quark condensates in medium plays a key role to understand the behavior of hadron masses and chiral symmetry restoration [1, 21. Recently, we calculated for the first time the second order response of the quark condensate to the chemical potential $\frac{d^{2}\langle\bar{q} q\rangle}{d \mu^{2}}$ at $\mu=$ 0 using lattice QCD [3] following the method in 四. It was found that the response is negative both below and above $T_{c}$. One of interesting results is that the response to the isoscalar chemical potential $\left(\mu_{S}=\mu_{u}=\mu_{d}\right)$ is almost the same as that to the isovector chemical potential $\left(\mu_{V}=\mu_{u}=-\mu_{d}\right)$, where $\mu_{u}\left(\mu_{d}\right)$ is the $u(d)$ quark chemical potential. It would be interesting if we can check this result within effective models of QCD. In this work we present an Nambu-Jona-Lasinio (NJL) model [5, 6] calculation of $\frac{d\langle\bar{q} q\rangle}{d \mu}$ and $\frac{d^{2}\langle\bar{q} q\rangle}{d \mu^{2}}$ at $\mu=0$ and compare our results with those from the lattice QCD simulations [3.

An SU(2) NJL model Lagrangian will be enough for that purpose. However, we are also interested in the responses of the s-quark condensate and a comparison with those of the u,d-quark condensates will be useful for future studies in the lattice calculations. Thus we used an SU(3) NJL model in our calculations and found that the effects of the flavor mixing are negligible. We present only the results for the $\mathrm{u}, \mathrm{d}$-quark condensates in this paper, and we will make the comparison in a forthcoming paper.

First, let us consider an SU(3) NJL model Lagrangian [7]:

$$
\begin{aligned}
L & =\bar{q}(i \gamma \cdot d-m) q+\frac{1}{2} g_{S} \sum_{a=0}^{8}\left[\left(\bar{q} \lambda_{a} q\right)^{2}+\left(\bar{q} i \lambda_{a} \gamma_{5} q\right)^{2}\right] \\
& +g_{D}\left[\operatorname{det} \bar{q}_{i}\left(1-\gamma_{5}\right) q_{j}+h . c .\right]
\end{aligned}
$$

where $\lambda_{a}$ are the Gell-Mann matrices and $m$ is a mass matrix for current quarks, $m=\operatorname{diag}\left(m_{u}, m_{d}, m_{s}\right)$. We take the following parameters in [7]:

$$
\Lambda=631.4 \mathrm{MeV}, g_{S} \Lambda^{2}=3.67, g_{D} \Lambda^{5}=-9.29
$$

${ }^{*}$ Deceased

$$
m_{u}=m_{d}=5.5 \mathrm{MeV}, m_{s}=135.7 \mathrm{MeV},
$$

where $\Lambda$ is the momentum cut-off. The third term in Eq.(11) is a reflection of the axial anomaly, and causes a mixing in flavors. For example, the constituent quark masses are given as follows.

$$
\begin{aligned}
M_{u} & =m_{u}-2 g_{S}\langle\bar{u} u\rangle-2 g_{D}\langle\bar{d} d\rangle\langle\bar{s} s\rangle, \\
M_{d} & =m_{d}-2 g_{S}\langle\bar{d} d\rangle-2 g_{D}\langle\bar{u} u\rangle\langle\bar{s} s\rangle, \\
M_{s} & =m_{s}-2 g_{S}\langle\bar{s} s\rangle-2 g_{D}\langle\bar{u} u\rangle\langle\bar{d} d\rangle,
\end{aligned}
$$

where $\langle\cdot\rangle$ means the statistical average. In this work we concentrate mostly on the Case II in [7], where only $g_{D}$ has a temperature dependence

$$
g_{D}(T)=g_{D}(T=0) \exp \left[-\left(T / T_{0}\right)^{2}\right],
$$

while other coupling constants and the cut-off are independent of $T$ and chemical potential (or density). Here, we set $T_{0}=0.1 \mathrm{GeV}$ taking into account the restoration of $U_{A}(1)$ symmetry as in [7].

In the mean-field approximation the above Lagrangian leads to the following gap equation $[7$ :

$$
Q_{i} \equiv 2 N_{c} \sum_{p}\left(\frac{-M_{i}}{E_{i p}} f\left(E_{i p}\right)\right)=\left\langle\bar{q}_{i} q_{i}\right\rangle
$$

where the index $i$ denotes the $u, d$, and $s$ quarks. $N_{c}$ is the number of colors and $M_{i}$ is the constituent quark mass, and $E_{i p}=\sqrt{M_{i}^{2}+p^{2}} . f\left(E_{i p}\right)=1-n_{i p}-\bar{n}_{i p}$, where $n_{i p}$ and $\bar{n}_{i p}$ are the distribution functions of the $i$ th quark and antiquark, respectively, i.e.,

$$
\begin{aligned}
n_{i p} & =\frac{1}{1+\exp \left(\left(E_{i p}-\mu_{i}\right) / T\right)}, \\
\bar{n}_{i p} & =\frac{1}{1+\exp \left(\left(E_{i p}+\mu_{i}\right) / T\right)} .
\end{aligned}
$$

The 1.h.s. of Eq.(5) is a function of $\langle\bar{u} u\rangle,\langle\bar{d} d\rangle,\langle\bar{s} s\rangle, \mu_{i}$, and $T$. Then, we obtain the first order response of quark condensates $\frac{d\langle\bar{u} u\rangle}{d \mu}, \frac{d\langle\bar{d} d\rangle}{d \mu}$, and $\frac{d\langle\bar{s} s\rangle}{d \mu}$ by differentiating both 
sides with respect to $\mu$ at a fixed $T$, i.e., we solve the following equations

$$
\begin{aligned}
\frac{d Q_{u}\left(\langle\bar{u} u\rangle,\langle\bar{d} d\rangle,\langle\bar{s} s\rangle, \mu_{u}\right)}{d \mu} & =\frac{d\langle\bar{u} u\rangle}{d \mu} \\
\frac{d Q_{d}\left(\langle\bar{u} u\rangle,\langle\bar{d} d\rangle,\langle\bar{s} s\rangle, \mu_{d}\right)}{d \mu} & =\frac{d\langle\bar{d} d\rangle}{d \mu} \\
\frac{d Q_{s}\left(\langle\bar{u} u\rangle,\langle\bar{d} d\rangle,\langle\bar{s} s\rangle, \mu_{s}\right)}{d \mu} & =\frac{d\langle\bar{s} s\rangle}{d \mu} .
\end{aligned}
$$

Here, we consider two types of $\mu$ following the notation in the lattice QCD simulations [3, 4]. One is the isoscalar chemical potential $\mu_{S}=\mu_{u}+\mu_{d}$, and the other is the isovector one $\mu_{V}=\mu_{u}-\mu_{d}$.

Let us comment on the definition of chemical potential used in the lattice calculations and ours. There will be no difference between the two definitions if we choose the same $\mathrm{u}$ - and d-quark mass. In the NJL model calculations we chose rather general definitions for the isoscalar and isovector chemical potentials, i.e., $\mu_{S}=\mu_{u}+\mu_{d}$ and $\mu_{V}$ $=\mu_{u}-\mu_{d}$ to show the quark mass dependence by taking different $\mathrm{u}$ - and d-quark masses.

Using Eq. (7) one can show that both $\frac{d\langle\bar{q} q\rangle}{d \mu_{S}}$ and $\frac{d\langle\bar{q} q\rangle}{d \mu_{V}}$ are zero at $\mu=0$. This is the same as the lattice QCD calculation [3]. At finite chemical potential, the absolute value of the quark condensate decreases with increasing chemical potential and the variation is proportional to the chemical potential in the present NJL model [8].

Next, consider the second order response $\frac{d^{2}\langle\bar{q} q\rangle}{d \mu^{2}}$. This is obtained by differentiating each equation in Eq. (7) with respect to $\mu_{S}\left(\right.$ or $\left.\mu_{V}\right)$ again. In Fig 10 we show $\frac{d^{2}|\langle\bar{q} q\rangle|}{d \mu^{2}}$ for the $u$ and $d$ quark condensates at $\mu=0$. Within the present NJL model the second order response to the isoscalar chemical potential $\mu_{S}$ is the same as that to the isovector chemical potential $\mu_{V}$. This is consistent with the result in [3]. In fact, there are a few different terms between the isoscalar and the isovector cases in the lattice QCD simulations. However, it is found that the contribution of those terms is negligible as shown in the figure 3 of Ref.[3].

In the lattice calculations the same $\mathrm{u}$ - and d-quark mass was taken [3]. In this paper, however, we would like to show the quark mass dependence of the response in the figure. Our main interest is to compare the responses below and above the Mott temperature depending on the quark mass. The present results may be useful for future lattice QCD simulations which use different $\mathrm{u}$ - and dquark masses.

We take $m_{u}=4 \mathrm{MeV}$ and $m_{d}=7 \mathrm{MeV}$ as well as $m_{u}$ $=m_{d}=5.5 \mathrm{MeV}$ to show the quark mass dependence of $\frac{d^{2}|\langle\bar{q} q\rangle|}{d \mu^{2}}$. Although the cut-off and the coupling constants should be modified, we use the same coupling constants and the cut-off for both cases and study the behavior of $\frac{d^{2}\langle\bar{q} q\rangle}{d \mu^{2}}$. Below the pion Mott temperature $T_{m_{\pi}}(\approx 148$ $\mathrm{MeV}$ in this work), the response of the $u\left(m_{u}=4 \mathrm{MeV}\right)$ quark condensate is larger than that of the $d\left(m_{d}=7\right.$ $\mathrm{MeV}$ ) quark condensate, while above the Mott temperature the behavior of the response is opposite (Here, $T_{m_{\pi}}$ is determined as a temperature at which the sum of the $u$ and $d$ constituent quark masses equals to the pion mass, i.e., $\left.M_{u}+M_{d}=m_{\pi}\right)$. This behavior results from the quark mass dependence of the first order response at finite chemical potential shown in Fig. 2, where we take $\mu_{u}$ $=\mu_{d}=0.02$ and $0.04 \mathrm{GeV}$, respectively. That behavior in Fig. 2 is not altered even in the Case I [7], where all the couplings and the cut-off are independent of temperature and chemical potential. For example, in the Case I and at $\mu=0.02 \mathrm{GeV}$, the pion Mott temperature is about $203 \mathrm{MeV}$ and the extremum is located at around $186 \mathrm{MeV}$.

In Fig. 1, we also present the relative variation $\frac{d^{2}\langle\bar{q} q\rangle}{d \mu^{2}} /\langle\bar{q} q\rangle$ at $\mu=0$ for comparison. Now, $\frac{d^{2}\langle\bar{q} q\rangle}{d \mu^{2}} /\langle\bar{q} q\rangle$ has the extremum at the Mott temperature and the (absolute value of) variation decreases with increasing the quark mass. We find similar behavior in the lattice data, although $\langle\bar{q} q\rangle$ is very small over $T_{c}$ in this case.

As we know, we can not detect a quark condensate itself in experiments. However, variations of quark condensates, such as shown in Fig. 2, are necessary to predict responses of hadron masses to the chemical potential, e.g., $\frac{d m}{d \mu}$ in [8]. A more detailed analysis on the extremum point and its effects on hadron masses is in progress.

In summary, we have calculated the first and second order responses of quark condensates to the chemical potential within the NJL model and found that they are consistent with those from the recent lattice QCD simulations.

\section{Acknowledgments}

We would like to thank Prof. Su H. Lee for valuable comments. Two of the authors (A.N. and T.T.) were supported by the Grant-in-Aide for Scientific Research by the Ministry of Education and Culture, Japan (11440080, 12554008,13135216 , and 13740164), and S.C. was supported by the Korean Ministry of Education under the BK21 program and in part by the Japan Society for the Promotion of Science (JSPS). This paper is based on a contribution to the Prof. Osamu Miyamura memorial symposium held at Institute for Nonlinear Sciences and Applied Mathematics (INSAM), Hiroshima University, Nov. 16-17, 2001. 

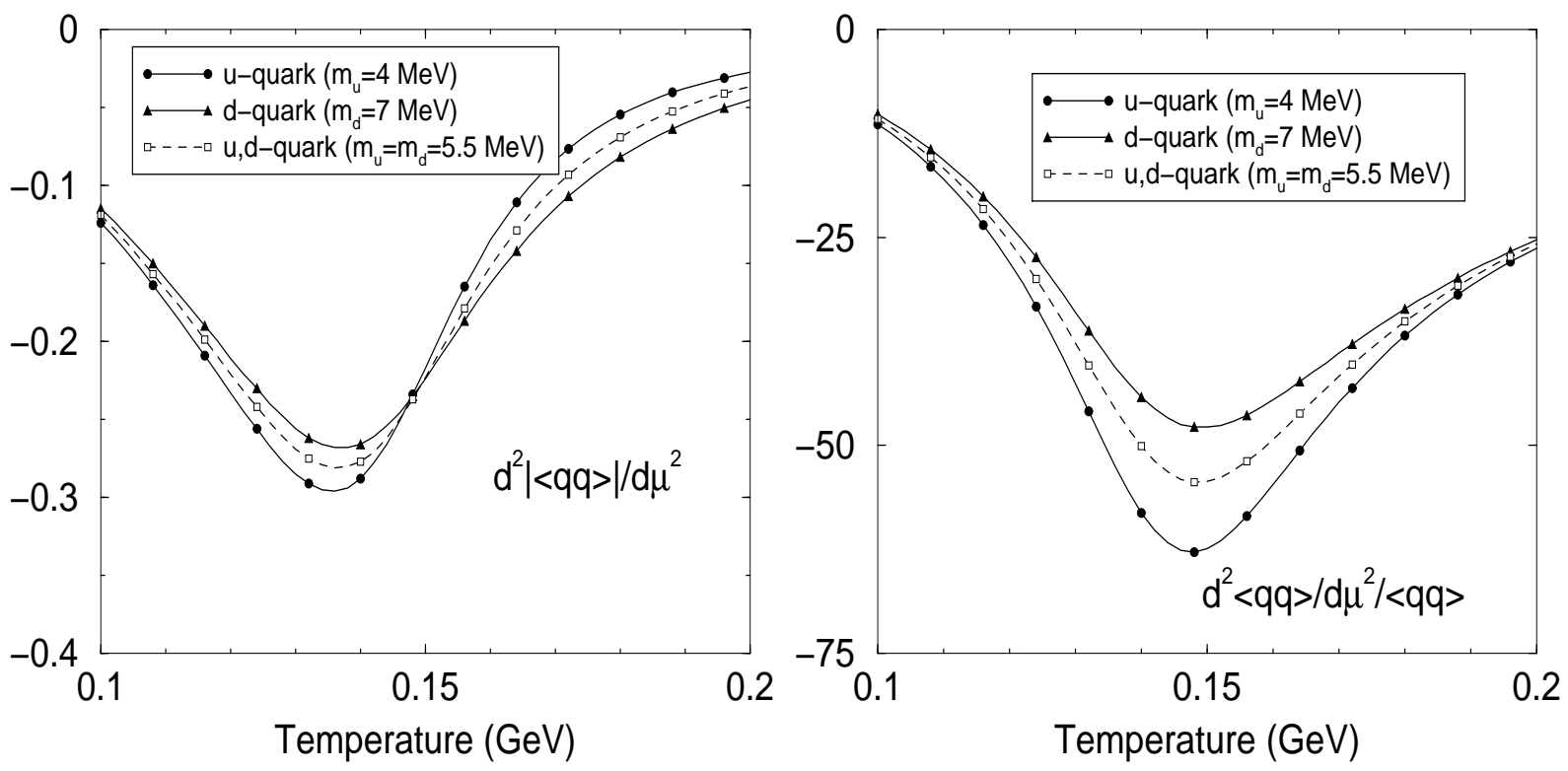

FIG. 1: The second order responses of quark condensates at $\mu=0: \frac{d^{2}\langle\bar{q} q\rangle}{d \mu^{2}}$ (left) and $\frac{d^{2}\langle\bar{q} q\rangle}{d \mu^{2}} /\langle\bar{q} q\rangle$ (right). Here, $\mu$ means both $\mu_{S}$ and $\mu_{V}$, i.e., $\frac{d^{2}\langle\bar{q} q\rangle}{d \mu_{S}^{2}}=\frac{d^{2}\langle\bar{q} q\rangle}{d \mu_{V}^{2}}$.

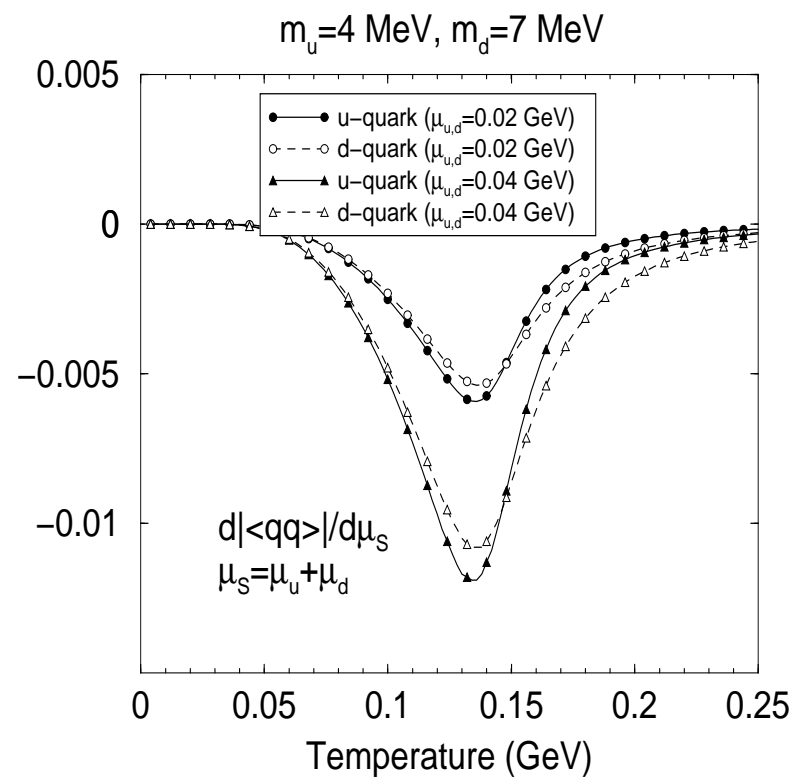

T. Hatsuda, Y. Koike, and Su H. Lee, Phys. Rev. D 47, 1225 (1993); Nucl. Phys. B 394, 221 (1993).

[3] QCD-TARO Collaboration, Nucl. Phys. B (Proc. Suppl.) 106, 462 (2002).

[4] QCD-TARO Collaboration, Phys. Rev. D 65, 054501 (2002).

[5] Y. Nambu and G. Jona-Lasinio, Phys. Rev. 122, 345 (1961); ibid. 124, 246 (1961).

[6] See, e.g., U. Vogl and W. Weise, Prog. Part. Nucl. Phys. 27, 195 (1991); S.P. Klevansky, Rev. Mod. Phys. 64, 649 (1992); and references therein.

[7] T. Hatsuda and T. Kunihiro, Phys. Rep. 247, 221 (1994).

[8] O. Miyamura and S. Choe, hep-ph/0105198; Nucl. Phys. B (Proc. Suppl.) 106, $474(2002)$.

FIG. 2: The first order responses of the $u$ and $d$ quark condensates at finite chemical potential, where $m_{u}=4 \mathrm{MeV}$ and $m_{d}=7 \mathrm{MeV}$. 